Journal of

Language and

JLD (PRINT) ISSN 2397-2637

Discrimination

JLD (ONLINE) ISSN 2397-2645

Review

\title{
Homing in on HATE: Critical Discourse Studies of Hate Speech, Discrimination and Inequality in the Digital Age Edited by G. Balirano and B. Hughes (2020) Napoli, Italy: Paolo Loffredo Editore srl, 267 pp.
}

Reviewed by Dr Mirko A. Demasi

KEYWORDS: CRITICAL DISCOURSE ANALYSIS; HATE SPEECH; ONLINE DISCOURSE; ONLINE HATE SPEECH

This is an edited volume looking at various aspects of hate speech online, through the lens of Critical Discourse Analysis (CDA) and its analytic subvariants. The edited chapters cover a diverse range of critical discourse analytic work, both in terms of theory and context. For theory, within the tradition of CDA, they cover analytic approaches such as CDA, Multimodal Critical Discourse Analysis (MCDA), corpus-based discourse analyses and more. The range of topics concerning online hate speech is varied - online fat shaming, ISIS propaganda online, Brexit hate speech on Twitter, and so forth.

The first section of the book focuses on the role of hate speech in the media. Balirano and Hughes demonstrate, in arguably the most analytically solid chapter in the book, how online (Twitter) hate speech pertaining to fat shaming can vary between cultures. Pitassi's analysis of Spanishspeaking radio in the USA demonstrates the reproduction and, at times, resistance of heteronormative values, particularly when directed towards women. It shows an interesting change in dynamics when women DJs are part of the radio programmes. Nisco focuses on the reproduction of hate speech directed at disabilities and how these, in the case of Katie Price and

\section{Affiliation}

Leeds School of Social Sciences, Leeds Beckett University email: mirko.demasi@leedsbeckett.ac.uk 
her son, intermingle with misogyny. The inference of cognition from the online comments is arguably theoretically and analytically less convincing, but that is down to my personal position, and the theoretical framework is in itself solid. The tension between freedom of speech and hate speech in Ben Shapiro's university talks, often aired online, is the focus of Zottola's analysis. A number of discursive strategies - such as othering, mockery and exerting control over the sessions while claiming to be open - are identified in the analysis. The chapter makes a solid point but suffers from not showing enough data. Such important, and still very valid, findings would warrant more data to showcase the findings in more detail. Russo takes us back to Twitter, using corpus analysis to demonstrate how risk news regarding Covid-19 are communicated and responded to. The use of hashtags demonstrate how Twitter users respond to fear appeals, discursively constructing in- and out-groups.

Rasulo looks at the visual and discursive organisation of the online newspapers run by the terrorist organisation, ISIS, particularly how the concept of cities, towns and villages feature in ISIS' arguments for conquest, destruction and dominance. The analysis is a convincing demonstration of how physical spaces, in this instance cities, can be discursively constructed to embody ideological positions. Demata and Zummo demonstrate the use of militarised language - in this instance Nigel Farage's tweet in 24/12/20 stating that 'the war is over' - in Brexit discourse. The analysis looks at how this tweet draws from wider historical and social contexts where militarised language is particularly relevant for a British audience. Additionally, several responses, affiliative and much less so, feature in the analysis and show how many of the responses will carry the initial militarised language into new discursive territory.

The second part of the book is dedicated to the role of hate speech regarding institutions and law. Cambria looks into responses to the Irish consultation into developing hate speech legislation. The analysis highlights the complex and contentious nature of what exactly counts as hate speech, as indicated by how people respond to the consultation. Pizzo carried out a corpus analysis of Facebook comments. The comments are from posts of four Italian, two left-wing and two right-wing, politicians involved in developing a new hate speech legislation in Italy. The analysis highlights the resistance of anti-hate speech ideals in online discourse. The chapters by Cambria and Pizzo also direct the gaze at another analytically fruitful avenue: people's reactions in policy consultations. These are important sites of analysis, on account that they may well have some notable influence on subsequent policy decisions. Sindoni analyses the discursive strategies of an anti-hate speech campaign group, Stop Funding

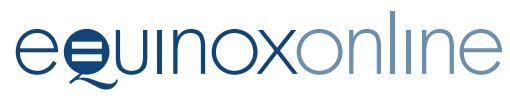


Hate. Carrying out a multimodal analysis of the campaign group's video, Sindoni demonstrated the visual-rhetorical construction of the anti-hate speech discourse. Particularly noting that the discursive moves mirror the discursive moves in hate speech. Taviano concludes the book with a chapter on how hate speech is perpetuated in British and Italian media. In addition, this chapter provides the most focused argument for strategies of combating hate speech. Highlighting the importance of the notion of translation, specifically away from a language of exclusion, Taviano argues for the importance of 'love speech' - a targeted new language (in some cases, new words too) that journalists, scholars and others alike are responsible for in order to combat hate speech.

The book is geared towards an audience already familiar with CDA. Though there are plenty of further resources for the reader to look into, some of the basic tenets of CDA would have been good to see. Overall, the analyses of the chapters are solid - though, in my opinion, some chapters could have shown more of their data. On account of this, for some chapters it was hard to get a full contextual sense of the data. The book suffers from the somewhat overly technical academic language that Billig (2013; 2019) warns of, though, it must be said, the overall accessible structure and length of chapters is commendable. What I particularly appreciated was the obviously systematic study of online discourse, whereby the analysis shows a number of important aspects of online hate speech.

This edited volume contributes to the study of online discourse and hate speech. As such it is a timely contribution to the study of online discourse as a site of paramount importance to the understanding of contemporary hate speech. Much of contemporary hate speech unfolds online, and it is crucial it is subject to academic scrutiny. This volume contributes to this from a CDA approach, particularly suited to the study of ideologies and oppression in discourse. While the book is set within one particular analytic tradition, it is, in addition, an important contribution to the broader qualitative studies of online hate speech. This book offers a clear, systematic, study of in situ online hate speech while showing a non-quantitative inroad to the study of the extremes of hate speech. This precedent will be a worthwhile legitimising of further research into the area of online hate speech. Indeed, while the analytic strength of individual chapters varies, the most significant contribution of this book is in putting forth, as a whole, a systematic and diverse study of online hate speech. Quantitative approaches tend to struggle to appreciate the nuance of hate speech, and it is time that qualitative approaches, in the broadest sense, are recognised as significant contributors to the empirical study and combating of hate speech. 
The volume is particularly suited for those who are interested in understanding contemporary hate speech, especially in its more common context of expression: the internet. However, the reader should bear in mind that this is set within one particular analytic tradition. Some familiarity with Critical Discourse Analysis is required of the reader. This book is suited for undergraduate, postgraduate students, as well as scholars.

\section{References}

Billig, M. (2013) Learn to Write Badly: How to Succeed in the Social Sciences. Cambridge: Cambridge University Press.

Billig, M. (2019) More Examples, Less Theory. Cambridge: Cambridge University Press. 